\title{
SELF-CARE MANAGEMENT AMONG ELDERLY PATIENTS WITH HYPERTENSION AND POOR ADHERENCE TO TREATMENT, LIVING IN RURAL THAILAND: A QUALITATIVE STUDY
}

\author{
Nanthakan Sungsuman Woodham ${ }^{1}$, Surasak Taneepanichskul', Ratana Samrongthong ${ }^{1}$, Nanta Auamkul $^{2}$ \\ ${ }^{1}$ College of Public Health Sciences, Chulalongkorn University, Bangkok, Thailand 10330 \\ ${ }^{2}$ The Planned Parenthood Association of Thailand (PPAT) under the Patronage of H.R.H the princess mother, \\ Bangkok, Thailand, 10900 \\ Correspondence: Ratana Somrongthong, Email: ratana.So@chula.ac.th
}

\begin{abstract}
Background: Hypertension is a common health problem among the elderly. Approximately $50 \%$ have poor adherence to their prescribed medication, which leads to uncontrolled blood pressure. This study was aimed to understand causes of poor adherence and gain knowledge of patient's self-management in their home.

Methods: In-depth interviews were conducted. Elderly hypertension patients with poor adherence to antihypertension medication and uncontrol blood pressure, between the ages of 60-79 were purposively selected to participate in this study. Home visits, including observation of patient's behavior in their home, were conducted. Content analysis was performed.

Results: A total of thirty respondents were interviewed of which 17 were female and 13 were male. All understood that hypertension is a chronic health problem but most of them were unaware that nonadherence to their hypertension medication treatment would lead to negative consequence to their health. Most respondents had negative beliefs toward hypertension treatment. The reasons for poor adherence are: 1) personal beliefs or habits, which include forgetfulness and negative belief that the medication will not control blood pressure; 2) side effects of medication and 3) negative perceptions influenced by the community. Research results showed that most participants were not taking their prescribed dosage. Additionally, expired medication was commonly found in their medication inventory. High sodium diets and no regular exercise was commonly reported.

Conclusion: Patients understood that hypertension is a lifelong condition but unaware of consequence of poor adherence. Personal reason and negative belief as well as wrong belief influenced by community. Forgetfulness and manage dosage of medication should be addressed when designing a strategy to improve medical adherence. Education on controlling hypertension and treatments should be addressed and incorporated into a holistic strategy to improve adherence to the patient's drug regimen.
\end{abstract}

Keywords: Hypertension, medication adherence, elderly, self-care management

\section{Introduction}

The World Health Organization (WHO) reported, globally 10 million adults suffer from hypertension, of which two thirds are in developing countries. In 2025, it is predicted that hypertension patients will increase to 1.56 billion(1).

Based on a study in 2008-2009, it is reported that $24.7 \%$ of the population suffered from hypertension, especially, among the elderly. It is expected that hypertension will have a growing impact on this age group(2).

Thailand is experiencing a rapid increase in elderly population 60 years and older. In 2000, this age group was 5.87 million and increased to 8.38 million in 2010 . The prediction is 12.39 million by 2020 . Based on this rapid increase, the health care system should plan at the all levels to make the health services suitable for elderly(3).
There are $41 \%$ of elderly have hypertension and almost $50 \%$ have poor adherence to their drug regiment and as such, have uncontrolled blood pressure. The study showed, almost half of them stopped taking antihypertension drugs due to no signs and symptoms. During the 5 to 10 years of follow-up, less than $40 \%$ of patients continued with their prescribed antihypertensive treatment. $(4,5)$.Other studies have reported numerous factors that affect adherence including lifestyle, psychological issues, health literacy, support systems and side effects of medications(6).

This study was conducted in Buengkan province, which is the newest province of Thailand and is considered a rural area. There is still lack of information and evidence with the elderly in this region, as it relates to self-care management in their home and adherence to their hypertension medication treatment. Additional 
information needs to be gathered among the elderly population in rural areas. The primary aim of this study was to gain a better understanding of elderly hypertension patient's perspective on living with hypertension and their knowledge on hypertension and treatment. Secondary, was to gain a deeper understanding of how the patients managed their medication at home. This information gathered from this study will be benefit in helping health care provider to develop strategies or programs to improve medical adherence from the root cause of problem among the elders.

\section{Methodology}

A qualitative approach using in-depth structured interviews, were conducted at the patient's home during home visits. Purposive sampling was adopted by interviewing 40 participants until a saturation of responses was reached. During the home visit, the dialogue included asking the patient to show how their medications are stored. Additionally, observations about diet were made based on leftover food. The inclusion criteria for these patients in this rural area of Thailand included:

1) Age between 60 - 79 years;

2) Diagnosed with hypertension for at least one year;

3) Made at least three hospital visits during the last six months with uncontrolled blood pressure; (systolic ? $140 \mathrm{~mm}$. Hg. diastolic? $90 \mathrm{~mm}$. Hg.)

4) Poor adherence to antihypertension medication, which was identified by pill count by health personnel.

5) Able to provide informed consent and willing to participant in the study.

Patients with severe health conditions were excluded from this study.

The interview lasted approximately $30-45$ minutes. The interview process was conducted by a researcher and a trained, degree-qualified research assistant. The interviewers have no relationship to the patients. An audio recording device was used to record the interviews as well as written notes taken based on observations of how the patients stored their medication.

The researcher announced and described general of study information at Buengkan Hospital. Patients who were interested to participate in the study were contacted face to face by the research assistants. Participants were asked to sign the consent form. Some patients who met inclusion criteria declined to participate in this study. Afew common reasons included:

1) Not have enough time to participate;

2) Not comfortable with a home visit;

3) Working in the rubber plantation at night and sleep during day time which not suitable for home visit and interview;

4) Some of research questions were too complicated to answer.

This study was reviewed and approved by
Chulalongkorn University ethical committee (141.1/60) and Beungkan Hospital ethical committee (BKHEC2018-01).

The recorded interviews and written notes are contained in the field notes of the study. The interview was conducted in the Isan dialect, which is common to Northeast Thailand, then transcribed into Thai, then coded and analyzed. In this process, data was collected on various characteristics and attributes of the participants to gather information that would provide better insights into their daily lives. Attributes of the participants included gender, marital status, living arrangements and whether the patient has a caretaker. An in-depth interview guide was developed by the researcher and reviewed by three experts. The guide included perceptions of living with hypertension, understanding hypertension and its treatment; reasons of not taking medication as prescribed, selfmanagement to control blood pressure, and storage of hypertension medication at home. A combination of methods was performed for triangulation. The researcher's observations documented in the field notes and information from hospital records. At the end of the interview, participants were asked to show how they manage their hypertension medication as well as explain how they take medication including number of pills, time each day they take medicine and what they do if they forget to take medication.

The study was analyzed by using a content analysis technique. Two trained independent research assistants analyzed the data. Data from the interviews was transcribed by research assistants and field notes from home visit observation session were analyzed.

\section{Results}

The 30 patients in the study consisted of 17 females and 13 males from different sociodemographic backgrounds. 19 were married, 6 were divorced, 4 widowers and 1 was single. All the patients can take care of themselves and only have a caretaker when they are sick. 24 patients have diabetes in addition to their hypertension. The themes from the interviews and observations include:

\section{1) Perception toward hypertension}

Many of the patients reported they understand that hypertension is a chronic lifelong disease, but don't believe that taking medication will deter serious health conditions. Some expressed they may consider discontinuing taking medication or stop taking it every day, to avoid health conditions in the future.

"I think taking medication will make me have more health conditions as I take medication for a long time. I am afraid my kidney will fail soon as I take too many medicines for a long time". (Female age 67)

However, most participants were aware that the doctor's appointment is important. Even though they do not want to take medicine as prescribed, they still think that coming to the hospital and following the appointment schedule is important. 
"I come to hospital and follow my appointment schedule every time at least to check my health condition but I do not believe that taking medicine every day will help control my blood pressure as it never gets lower". (Male age 70)

\section{2) Knowledge of hypertension and its treatment}

Most participants do not know causes of hypertension. Most believe that older persons tend to have hypertension.

"I do not know the cause of it but people who get older are more likely to have hypertension than young person." (female age 65)

When asked about what they could do to control hypertension, all knew that avoiding salty and oily foods was important for controlling hypertension as well as taking their medication as prescribed. However, many have negative perceptions of taking their medication.

"Avoid salty and oily food and take medicine. I had heard people die from kidney failure from taking too much medicine, so I think maybe taking medicine is ok, but not as many as we are given. (Female age 68)

When asked about the name of their medication, none of participants knew the name of the medication but, generally, they can identify each medicine and what it treats. All of them remember the colors and shape of the package and pills and based on this, which medicine to take. This is method is used for all their medications.

"I don't know the medicine's name, but I know what it's for and I can remember color, shape and package of medicine which that tells me if that medicine is for hypertension." (Male age 69)

When asked how they receive information regarding hypertension and other chronic diseases, all commented they receive information from the hospital and health promoting hospital.

"While waiting to see doctor in hospital there were nurses who give information about hypertension and diabetes to us." (Female age 74)

\section{3) Reason of poor adherence to hypertension medication}

More than half of participants reported that they are taking medication per their prescription but from the pill count results and observations by the researcher and research assistant, it shows that they are in poor adherence to their treatment. Reasons for poor adherence were based on both personal beliefs and perceptions regarding antihypertension medication. Personal beliefs included; not following prescription, take wrong dosage, negative beliefs about medication and forgetfulness. Medication and disease related reasons included avoiding side effects and no signs or symptoms.

\section{A. Do not follow prescription.}

"I remember all my medicine, what and when to take because I took this medicine for more 5 years". (Female age 70)

With some patients, the researcher asked to see the medication package and the prescription written on the package. After inspection, many patients were taking the wrong dosage even though they mentioned they were following the prescription.

B. Forgetfulness. All of patients reported that they sometimes forget to take hypertension medicine.

"When I go to the farm or go to the temple in the morning, I take breakfast there, but my medicine was at home and that make me miss taking that dose of medicine" (Male age 64)

C. Belief that taking many medications would cause other serious health problems.

"We think that taking hypertension medicine for a long time will damage kidney and liver which is the reason why people have diabetes after hypertension so that's why I don't take medicine every day". (Female age 67)

D. Take wrong dosage due to misunderstanding the dosage instructions.

"I cut that big white medicine into four pieces and I took one piece the rest I throw away. Some months I finish all the medicine in 10 days. (Female age 62)

E. Are confused or uncomfortable when packaging is changed.

"Doctor gave me new medicine. I don't see reason to take more medicine because I still have many left at home. (Male age 69).

F. Wanting to avoid potential side effects of medicine.

"When I take medicine, I feel tired, cough a lot and cannot sleep at night so I stop taking medicine." (Female age 68)

G. No signs and symptoms. Many participants mentioned, there have no signs and symptoms which leads them to believe there is no reason to continue taking medicine.

"I don't feel like I need to take medicine because I don't have any signs or symptoms. I don't have headache or anything. I don't see reason why I need to take medicine." (Male age 66)

\section{4) Self-care management at home of hypertension patients}

Most participants take care of themselves at home. They understand that to control their blood pressure, just medicine is not enough. It also requires controlling diet, stress and exercise.

"The doctor told us to take medicine and don't eat salty and oily foods. I tried to follow the advice, but still every time I check my blood pressure it is still high". (Female age 67)

Most patients expressed they do not consume salty or oily foods, however the researcher discovered that while the patients avoid salt, the seasonings they use are high in monosodium glutamate.

"My daughter is concerned that I have hypertension, so she does not put much salt in the food. She mostly uses fish sauce and roddee." (Female age 69)

All participants mentioned they do not exercise regularly. "I don't have exercise regularly, but I walk to temple every morning. From my house to temple it takes about 15 
minutes." (Female age 64)

\section{5) Patients manage and store their hypertension medication at home}

All participants managed their own medication. All participants store their medication in bags that contain many other prescription drugs. Expired medication was commonly found being stored. New and old medicines were stored together in one place.

"I manage my own medicine and take it based on my routine as I have taken medicine for so long. I know better than anyone what to take." (Female age 60)

In addition, most patients used a simple clear plastic bag for storage of their medicines and some stored their medicine is hot areas. None of the participants showed any organization of their medications. None of the patients were aware to check the expiry dates.

"I do not know how to check and never checked expire date. After I received medicine from hospital I put them all in the same place". (male age 61)

\section{Discussion}

Regarding the perception of living with hypertension, participants understand that it is a chronic long-life disease, but they have little knowledge about hypertension and treatment. This study was in line with study conducted in rural Thailand, that showed awareness about hypertension, treatment and controlling of hypertension, was low. There is an urgent need to provide knowledge about hypertension and improve the awareness among the population in the rural areas(7).

The results showed patients take wrong dosage or wrong medicine. A few reasons given for this were relying on their memory as part of their routine for taking medicine for long period of time; also, for patients who are required to take partial doses, a misunderstanding of cutting the tablets and only taking a portion of medicine. Additionally, prescription changes confuse some patients as it's different in size and shape of the previous medication. Forgetfulness was common among patients. When there are no signs or symptoms, some patients believe their medicine is no longer required. By not taking their medicine, they believe they will avoid potential side effects of the medicine. Our findings are similar to a previous a qualitative study, where reasons for non-adherence were misunderstanding the conditions of the medicine and general disapproval of medication. However, most continued taking the medicine to facilitate their daily life or minimize the side effects of medicine(8). The interview results are in line with study regarding hypertension treatment where adherence was found to be better among those patients who are only taking one medication per day, who never change their medication regimen and those who continue visit the same doctor over time(9).

Other studies published similar key risk factors associated with poor adherence including: lack of a medication administration routine, therapeutic duplication, hoarding, confusion between generic and trade names, multiple prescribers, discontinued medication and multiple storage locations. Forgetfulness, lack of effectiveness, adverse side effects and patient's general dissatisfaction with the medicine have also shown to be contributors to adherence levels. These studies also align with our proposition that there are several barriers for good adherence among elderly that need to be addressed(911). In addition, improved methods to provide accurate education to patients and the community on the nature of hypertension and its treatment, may be helpful to improving adherence among this group of patients.

There are several published works regarding the management of hypertension at home among elderly patients. This literature supports the idea that a controlled diet, exercise and adherence to medication will assist in controlling blood pressure(12-15). Dietary habits among elderly in the rural areas in northeast of Thailand found that salty food is common in their $\operatorname{diet}(16)$. The interviews showed that there is still a lack of information provided to this group of patients as most of them misunderstand what constitutes a salty diet where they only focus on salt intake but no other salt products such as seasoning ingredients that contain high levels of monosodium glutamate.

From the interviews, many patients indicated that they were influenced by friends or others in the community to discontinue their medications because the perception is that taking this medicine over a long period of time could potentially lead to additional health problems. Therefore, the comprehensive education program for the community should be implemented. Providing education can't be the only solution to increase awareness of hypertension. Continued dialogue at the outpatient clinic for each visit, home visits by healthcare workers and family-based discussions will contribute to higher rates of adherence from the patients $(17,18)$.

There are several studies published regarding the management of medications, but not how the elderly store their medications at home. From our findings, results show that the elderly manage their own medication with little or no support from family members. Our findings suggested that management of medication at home by elderly patients need supervision from family to be able to adhere to their prescription. Studies reported that multiple storage locations of medication are a contributor to poor adherence $(19,20)$. Our observation found that most patients stored their medications in a single place but with no organization. Lack of organization was a contributor to taking wrong medicine or expired medicine. Storage and organizing medicine is an urgent need to be addressed as patients taking expired medicine may experience negative health outcomes. Providing education to the patients alone is not enough. It needs to be innovative and combined with strategies for monitoring, to determine whether the patient is taking their medication as per the prescription $(4,21)$. 


\section{Conclusion}

Study concluded that the key reasons to poor adherence include; personal beliefs or habits, side effects of medication and negative beliefs influenced by the community. Forgetfulness, organization of medication, and storage should be addressed when design strategies to improve medical adherence among elders. Health care providers should pay attention in providing accurate information to patients and community. Education on controlling hypertension and treatments should be addressed and incorporated into a holistic strategy to improve adherence to the patient's drug regimen.

\section{Acknowledgments}

This study was supported by THE 100 THAnniversary of Chulalongkorn University Scholarship.

\section{References}

1. World Health Organization. A global brief on hypertension: silent killer, global public health crisis. Switzerland: World Health Organization, 2013.

2. Gakidou E, Mallinger L, Abbott-Klafter J, Guerrero R, Villalpando S, Ridaura RL, et al. Management of diabetes and associated cardiovascular risk factors in seven countries: a comparison of data from national health examination surveys. Bulletin of the World Health Organization. 2011;89(3):172-83.

3. Knodel J, TEERAWICHITCHAINAN BP, Prachuabmoh V, Pothisiri $W$. The situation of Thailand's older population: An update based on the 2014 Survey of Older Persons in Thailand. 2015.

4. Kamran A, Ahari SS, Biria M, Malpour A, Heydari $\mathrm{H}$. Determinants of patient's adherence to hypertension medications: application of health belief model among rural patients. Annals of medical and health sciences research. 2014;4(6):922-7.

5. Abegaz TM, Tefera YG, Befekadu Abebe T. Target Organ Damage and the Long Term Effect of Nonadherence to Clinical Practice Guidelines in Patients with Hypertension: A Retrospective Cohort Study. International journal of hypertension. 2017;2017.

6. Wang W, Lau Y, Loo A, Chow A, Thompson DR. Medication adherence and its associated factors among Chinese community-dwelling older adults with hypertension. Heart \& Lung: The Journal of Acute and Critical Care. 2014;43(4):278-83.

7. Udompittayason W, Boonyasopun U, Songwathana P. Perspectives on Hypertension among Thai-Melayu Elderly in a Province of Southern Thailand: An Ethnographic Study. ?? ?? ? ? ? ? ? ? ? ? ? ? ?? ? Songklanagarind Journal of Nursing. 2015;35(2):45-60.
8. Kassavou A, Sutton S. Reasons for nonadherence to cardiometabolic medications, and acceptability of an interactive voice response intervention in patients with hypertension and type 2 diabetes in primary care: a qualitative study. BMJ open. 2017;7(8):e015597.

9. Arts DL, Voncken AG, Medlock S, Abu-HannaA, van Weert HC. Reasons for intentional guideline non-adherence: a systematic review. International journal of medical informatics. 2016;89:55-62.

10. Náfrádi L, Galimberti E, Nakamoto K, Schulz PJ. Intentional and unintentional medication nonadherence in hypertension: the role of health literacy, empowerment and medication beliefs. Journal of public health research. 2016;5(3).

11. Herrera PA, Moncada L, Defey D. Understanding non-adherence from the inside: hypertensive patients' motivations for adhering and not adhering. Qualitative health research. 2017;27(7):1023-34.

12. Nyaaba G, de-Graft Aikins A, Stronks K, Agyemang C, Masana L. 5.10-P9 Exploring selfmanagement practices of people living with hypertension: a comparative study of Ghanaian migrants living in Amsterdam and Ghanaians residing in Ghana. The European Journal of Public Health. 2018;28(suppl_1):cky048. 195.

13. Park E, Kim J. The Impact of a Nurse? Led Home Visitation Program on Hypertension Self? Management among Older Community?Dwelling Koreans. Public Health Nursing. 2016;33(1):42-52.

14. Hu K, Wu S-y, Sun K-g, Zhang X-x, He C, Zhao $\mathrm{Y}-\mathrm{y}$, et al. The Association Between SelfManagement Behaviors and Medication Adherence in Patients With Hypertension. American Journal of Hypertension. 2018;31:1.

15. Polonia J, Martins L, Pinto F, Nazare J. Prevalence, awareness, treatment and control of hypertension and salt intake in Portugal: changes over a decade. The PHYSA study. Journal of hypertension. 2014;32(6):1211-21.

16. Amarra MS, Khor GL. Sodium Consumption in Southeast Asia: An Updated Review of Intake Levels and Dietary Sources in Six Countries. Preventive Nutrition: Springer; 2015. p. 765-92.

17. Conn VS, Ruppar TM, Chase J-AD, Enriquez M, Cooper PS. Interventions to improve medication adherence in hypertensive patients: systematic review and meta-analysis. Current hypertension reports. 2015;17(12):94.

18. Tsuyuki RT, Houle SK, Charrois TL, Kolber MR, Rosenthal MM, Lewanczuk $R$, et al. A randomized trial of the effect of pharmacist prescribing on improving blood pressure in the community: the Alberta clinical trial in optimizing hypertension (RXACTION). Circulation. 
2015:CIRCULATIONAHA. 115.015464.

19. Ewen S, Baumgarten T, Rettig-Ewen V, Mahfoud F, Griese-Mammen N, Schulz M, et al. Analyses of drugs stored at home by elderly patients with chronic heart failure. Clinical Research in Cardiology. 2015;104(4):320-7.

20. Kennedy-Hendricks A, Gielen A, McDonald E, McGinty EE, Shields W, Barry CL. Medication sharing, storage, and disposal practices for opioid medications among US adults. JAMA internal medicine. 2016;176(7):1027-9.

21. Costa E, Giardini A, Savin M, Menditto E, Lehane E, Laosa O, et al. Interventional tools to improve medication adherence: review of literature. Patient preference and adherence. 2015;9:1303. 